'E Griesmaier, 'A Posod, 'MJ Gross, 'V Neubauer, 'K Wegleiter, ${ }^{2} \mathrm{M}$ Hermann, 'M Urbanek, ${ }^{1,3} \mathrm{M}$ Keller, 'U KiechlKohlendorfer. 'Department of Paediatrics IV, Division of Neonatology; '2Department of Anaesthesiology, Innsbruck Medical University, Innsbruck, Austria; ${ }^{3}$ Department of Paediatrics, Children's Hospital Passau, Passau, Germany

Background Excessive glutamate release followed by N-methyl-Daspartate receptor (NMDAR) activation displays an important cascade in the pathophysiology of perinatal brain injury. We have previously shown that dextromethorphan, a low-affinity NMDAR antagonist, is neuroprotective in an animal model of neonatal excitotoxic brain injury. Of interest, dextromethorphan also shows agonistic properties at the sigma-1 receptor $(\sigma 1 \mathrm{R})$. Sigma- 1 agonists have given beneficial results in animal models of adult brain injury. Aim of the study To evaluate the effect of the selective $\sigma 1 \mathrm{R}$ agonist 2-(4-morpholinethyl) 1-phenylcyclohexanecarboxylate (PRE084) in neonatal excitotoxic brain injury.

Results A single intraperitoneal injection of $0.1 \mu \mathrm{g} / \mathrm{g}$ (low dose) or $10 \mu \mathrm{g} / \mathrm{g}$ (high dose) bodyweight (bw) PRE-084, given $1 \mathrm{~h}$ after the excitotoxic insult, significantly reduced lesion size in cortical gray matter $24 \mathrm{~h}$ and $120 \mathrm{~h}$ after the insult. Repetitive injections of 0.1 $\mu \mathrm{g} / \mathrm{g}$ PRE-084 proved to be equally effective. PRE-084 treatment resulted in a decrease in cell death indicated by reduced TUNEL positivity and caspase- 3 activation. PRE-084 reduced the number of isolectin B4-positive, activated microglial cells. Quantitative realtime PCR analysis showed no effect on $\sigma 1 \mathrm{R}$ gene expression at 1,4 , $8,12,24$ and $48 \mathrm{~h}$ after intracranial ibotenate injection compared to healthy controls. In vitro PRE-084 protected against glutamateinduced morphological and functional changes in primary hippocampal neurons.

Conclusion We demonstrate that systemic treatment with the highly selective $\sigma 1 \mathrm{R}$ agonist PRE-084 protects against NMDARmediated excitotoxic brain damage.

\section{CIRCULATING PROGENITOR CELLS IN PRETERM NEONATES WITH CNS INJURY - A PRELIMINARY REPORT}

doi:10.1136/archdischild-2012-302724.0300

${ }^{1} \mathrm{~N}$ Efstathiou, ${ }^{2} \mathrm{G}$ Kyriazis, ${ }^{3} \mathrm{D}$ Bougiouklis, 'V Drossou, 'V Soumbasi. ${ }^{1} 1$ st Neonatal Department, Ippokratio General Hospital; ${ }^{2}$ Immunology Department, Papanikolaou General Hospital, Aristotele University of Thessaloniki; ${ }^{3}$ Biologist, Specialist in Flow Cytometry, Thessaloníki, Greece

Background and Aims The assumption that the circulating progenitor cells participate in a chemotactic way in the endogenous regeneration effort after damage of body tissues is controversial and the exact type of the cells involved remains unknown, especially when referring to damages of the Central Nervous System (CNS). We assume that preterm neonates who undergo CNS injury respond in a similar way, and we investigate the progenitor cell populations that might be related to the devastating event.

Methods 23 preterm newborns were enrolled (gestation age $\leq 32$ weeks). 10 of them undergo severe perinatal stress with metabolic acidosis and developed CNS injury (IVH III or higher, PVL or infarct), whereas 13 of them were assumed as controls with no obvious CNS injury. Peripheral blood was collected at days 1, 3, 9, 18 and 45 after birth and analyzed using flow cytometry. Cell populations of interest were EPCs (Endothelial progenitor cells, CD34+/CD133+/CD184+), HSCs (Hematopoietic stem cells, CD34+/CD184+/CD45+) and VSELs (Very Small Embryonic-Like SCs, CD34+/CD184+/CD45-).

Results EPCs were significantly increased in the group with CNS injury at days 1.9 and 18 and VSELs were marginally increased at day 1 and significantly at day 9. HSCs showed no specific variation

Conclusion Circulating progenitor cells seem to play a role in the endogenous regeneration effort. Enhancing this effort might prove to be a good therapeutic practice in the future, whereas delineation of the timeframe of this effort would be essential. Larger studies are needed, as well as correlation with chemoattractants and longterm outcome is necessary.

\section{HYPERACTIVITY AND DISTURBED MOTOR COORDINATION IN ADOLESCENT MICE AFTER NEONATAL EXPSOURE TO HYPEROXIA}

doi:10.1136/archdischild-2012-302724.0301

${ }^{1} \mathrm{~T}$ Schmitz, ${ }^{1} \mathrm{~S}$ Endesfelder, ${ }^{2} \mathrm{MC}$ Reinert, ${ }^{3} \mathrm{~S}$ Müller, ${ }^{2} \mathrm{D}$ Liebetanz, ${ }^{1} \mathrm{C}$ Bührer. ${ }^{1}$ Department of Neonatology, Charité Universitätsmedizin Berlin, Berlin; ${ }^{2}$ Neurophysiology, Georg-August-Universität, Göttingen; ${ }^{3} B e r l i n$ Center for Stroke Research, Charité Universitätsmedizin Berlin, Berlin, Germany

Objective A small fraction of children born very preterm have overt cerebral palsy but many of them display subtle deficits in motor coordination, balance, and attention-deficit/hyperactivity disorder-like behavior. In the immature brain, the sudden increase of oxygen tension after birth amounts to hyperoxia, and experimental hyperoxia causes wide-ranging cerebral changes in neonatal rodents. Methods Newborn mice were exposed to $48 \mathrm{~h}$ of hyperoxia $(80 \%$ $\mathrm{O}_{2}$ ) from P6 to P8, and motor activity in running wheels was tested starting at adolescent age P30. Thereafter, from P44 to P53, regular wheels were replaced by complex wheels with variable crossbar positions to assess motor coordination. To determine white matter diffusivity, MRI with diffusion tensor imaging was performed in the corpus callosum in mice after hyperoxia at ages P30 and P53 in comparison to control animals always kept in room air.

Results Mice after neonatal hyperoxia had significantly higher values for maximum velocity and mean velocity in regular wheels than did control animals $(\mathrm{P}<0.05)$. In contrast, the motor challenge of the complex running wheels caused a greated decrease of maximum velocity in mice previously exposed to hyperoxia than in controls $(\mathrm{P}<0.05)$. Lower fractional anisotropy and higher radial diffusivity were observed in the corpus callosum of P30 and P53 mice after neonatal hyperoxia compared to control mice.

Interpretation Newborn mice exposed to hyperoxia display hyperactivity, motor coordination deficits, and impaired white matter diffusivity at adolescent and young adult ages.

\section{SEIZURE BURDEN AND NEUROBEHAVIORAL SCORES AFTER THERAPEUTIC HYPOTHERMIA IN THE NEWBORN PIGLET}

doi:10.1136/archdischild-2012-302724.0302

${ }^{1} \mathrm{X}$ Fan, ${ }^{2} \mathrm{C}$ Peeters-Scholte, ${ }^{1} \mathrm{~N}$ Ward, ${ }^{1} \mathrm{~PB}$ Colditz, ${ }^{1} \mathrm{ST}$ Bjorkman. 'Perinatal Research Centre, UQ Centre for Clinical Research, The University of Queensland, Brisbane, OLD, Australia; ${ }^{2}$ Neurophyxia B.V, Den Bosch, The Netherlands

Background Therapeutic hypothermia (TH) is standard of care in newborns with hypoxic-ischemic encephalopathy (HIE). Although the predictive value of amplitude-integrated EEG (aEEG) after HIE has been studied, the predictive value of aEEG during TH remains to be established.

Aim To study aEEG characteristics and timing of recovery of neurobehavior in a newborn piglet model of HIE following TH.

Methods Newborn piglets $(\mathrm{N}=14)$ were subjected to $30 \mathrm{~min}$ hypoxia-ischemia and survived to $72 \mathrm{~h}$. Animals were randomly assigned to hypothermia $(\mathrm{N}=8)$ or normothermia for $24 \mathrm{~h}$ after hypoxia-ischemia $(\mathrm{N}=6)$. aEEG was continuously recorded until $\sim 40 \mathrm{~h}$ post-insult and at 48 and $72 \mathrm{~h}$ post-insult. Background pattern aEEG and presence of seizures were analysed. Neurobehavior was scored from 40 until $72 \mathrm{~h}$ post-insult.

Results In hypothermic piglets aEEG background pattern recovered to continuous low voltage (CLV) within $2 \mathrm{~h}$ post-insult until $36 \mathrm{~h}$ post-insult. Normothermic piglets recovered within $2 \mathrm{~h}$ postinsult to continuous normal voltage (CNV) until 36h, where there was a decrease in background pattern to CLV. aEEG recovered to $\mathrm{CNV}$ in both groups by $72 \mathrm{~h}$ post-insult. Seizures were recorded in 
$50 \%$ of hypothermic piglets c.f. $83 \%$ in normothermic piglets. At $48 \mathrm{~h}$ post insult both groups showed a maximum of epileptic activity. The neurobehavioral score in the normothermic piglets showed an earlier return to baseline compared to the hypothermic piglets.

Conclusions Electrographic seizure burden was decreased following TH. aEEG background pattern and neurobehaviour score recovered earlier in normothermic piglets, suggesting that in the clinical situation conclusions based on aEEG and neurological examination should not be performed too early.

\section{HIGH DOSE INTRAVENOUS MELATONIN FOR AUGMENTATION OF HYPOTHERMIC NEUROPROTECTION LEADS TO HYPOTENSION}

doi:10.1136/archdischild-2012-302724.0303

M Ezzati, S Faulkner, K Broad, NJ Robertson. Institute for Women's Health, University College London, London, UK

Background Therapeutic hypothermia provides neuroprotection in infants with moderate to severe neonatal encephalopathy, however further treatments are necessary as hypothermic neuroprotection is not absolute.

Aim To assess the effect on mean arterial blood pressure of high dose intravenous melatonin $(20,10,5 \mathrm{mg} / \mathrm{kg})$ administered $10 \mathrm{~min}$ utes after hypoxia-ischemia.

Methods Male piglets underwent a hypoxic-ischemic insult and were then commenced on different doses of intravenous melatonin. Physiological measures such as mean arterial blood pressure and heart rate were measured. Hypothermia (core temperature $33.5^{\circ} \mathrm{C}$ ) was induced at 2-26h after hypoxia-ischemia.

Results $20 \mathrm{mg} / \mathrm{kg}$ melatonin induced a rapid reduction in blood pressure beginning at 1 hour following its administration (1-3h post administration; mean arterial blood pressure was reduced from 60 to $24 \mathrm{~mm} \mathrm{Hg}$. $10 \mathrm{mg} / \mathrm{kg}$ induced a gradual reduction in blood pressure at 1 hour post administration (1-4h post administration; mean arterial blood pressure was reduced from 55 to $31 \mathrm{~mm} \mathrm{Hg}$ ). However treatment with either 0 or $5 \mathrm{mg} / \mathrm{kg}$ melatonin had no effect on mean arterial blood pressure.

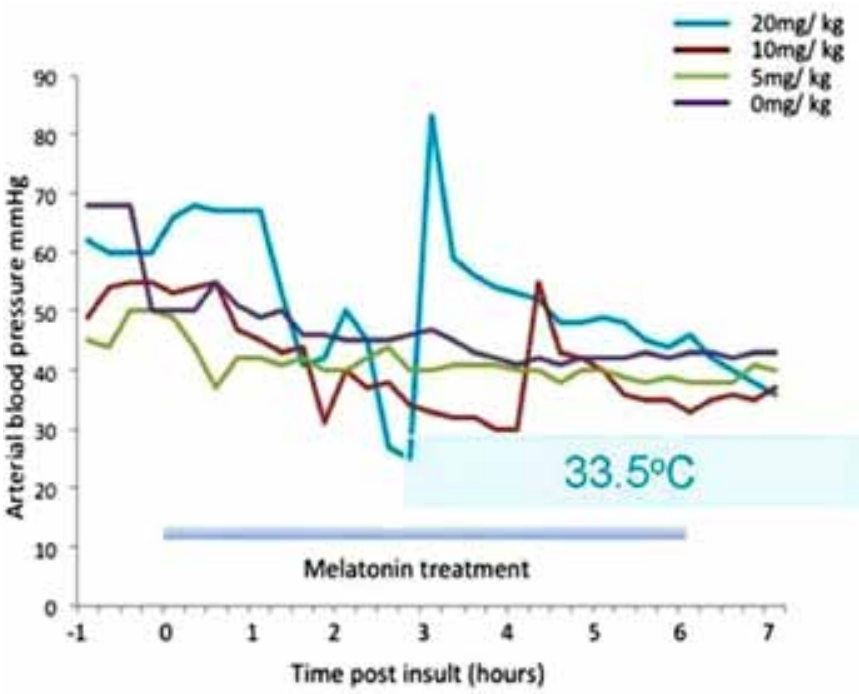

Abstract 303 Figure 1 Effect of different Melatonin doses on mean BP

Conclusion Intravenous doses of melatonin $(>5 \mathrm{mg} / \mathrm{kg}$ ) lead to hypotension following a hypoxic-ischemic insult when combined with hypothermia. Future pre-clinical studies of augmented hypothermic neuroprotection should be conducted using melatonin doses of $5 \mathrm{mg} / \mathrm{kg}$ or less.

\section{MINOCYCLINE ATTENUATES INJURY OF OLIGODENDROGLIAL PRECURSOR CELLS CAUSED BY OXYGEN-GLUCOSE-DEPRIVATION}

doi:10.1136/archdischild-2012-302724.0304

'T Schmitz, 'S Endesfelder, 'I Zaak, ${ }^{2}$ LJ Chew, ${ }^{1} \mathrm{C}$ Bührer. 'Department of Neonatology, Charité Universitätsmedizin Berlin, Berlin, Germany; ${ }^{2}$ Center for Neuroscience Research, Children's National Medical Center, Washington, DC, USA

Background Oxygen-glucose deprivation (OGD) is a widely used in vitro model for ischemic brain injury, which leads to cell death. Prevention and attenuation of brain injury by the tetracycline-antibiotic minocycline has been largely attributed to suppression of microglial activation.

Methods Using mono-cultures of rat oligodendroglial precursor cells (OPC) exposed to oxygen-glucose deprivation (OGD), we investigated direct effects of minocycline on survival, proliferation, and maturation of oligodendroglial lineage cells.

Results OGD for $2 \mathrm{~h}$ decreased the number of A2B $5+$ cells and the amount of proliferating Ki67+ A2B $5+$ cells by $50 \%$ which was both attenuated by minocycline in a dose-dependent fashion. The reduced numbers of $\mathrm{O} 4+$ cells at $72 \mathrm{~h}$ and of $\mathrm{O} 1+$ cells at $120 \mathrm{~h}$ after OGD were partially restored by minocycline. In OPC, OGD caused an increase of reactive oxygen species (ROS) production and of TUNEL-positive cell numbers which was abolished by minocycline, possibly via induction of superoxide dismutase. Minocycline also prevented OGD-induced downregulation of the expression of the transcription factors Sox10 and Olig2, and of the maturation markers 2'3' cyclic nucleotide phosphodiesterase (CNP) and myelin basic protein (MBP)

Conclusion The results demonstate that minocycline exerts direct protective actions on oligodendroglial lineage cells.

\section{DOPAMINE REDUCES WHITE MATTER INJURY IN HYPOXIC- ISCHAEMIA IN THE PRETERM LAMB}

doi:10.1136/archdischild-2012-302724.0305

F Wong, K Cassimally, A Azhan, T Samarasinghe, I Nitsos, A Walker, D Walker. The Ritchie Centre, Monash University, Melbourne, VIC, Australia

Background Dopamine is frequently used as inotropic agent in preterm infants. Its cardiovascular actions, as well as effects on neurovascular interactions may be neuroprotective during hypoxicischaemic events. Using a preterm lamb model we aimed to test the impact of intravenous dopamine on hypoxic-ischaemic brain injury. Method Nine fetal lambs (91-93d gestation) were instrumented with catheters in carotid artery and jugular vein, and an umbilical cord occluder. Four days after surgery, intravenous dopamine (DA, $10 \mu \mathrm{g} / \mathrm{kg} / \mathrm{min}, \mathrm{n}=5$ ) (or saline, $\mathrm{n}=4$ ) was commenced. then a hypoxic-ischaemic insult was induced with umbilical cord occlusion for 25 mins. Infusions were continued for another 72 hours before euthanasia. Fetal brains were collected for immunohistopathology. Results Dopamine infusion increased fetal heart rate $(184 \pm 1$ to $203 \pm 1 \mathrm{bpm}, \mathrm{p}<0.05)$ while arterial pressure was unchanged. Three animals in the DA group showed tachycardic response to cord occlusion, while the other two animals showed bradycardic response similar to the saline group. In the periventricular white matter, the saline group had higher number of microglia (lectin positive) than the DA group ( $10 \pm 3$ vs $6 \pm 2$ per $0.04 \mathrm{~mm}^{2}, \mathrm{p}<0.05$ ) The saline group tended to have shorter myelinated fibre lengths (CNPase) compared with the DA group $(15.0 \pm 2.0$ vs $18.4 \pm 5.7 \mu \mathrm{m}$ respectively, $\mathrm{p}=\mathrm{ns}$ ). No histological differences were evident between DA animals exhibiting a tachycardic or bradycardic response during cord occlusion.

Conclusions Intravenous dopamine reduces hypoxic-ischaemic white matter injury in preterm lambs, independent of the cardiovascular response during the hypoxic-ischaemia. 